\title{
Goldilocks Catalysts: Computational Insights into the Role of the 3,3' Substituents on the Selectivity of BINOL-Derived Phosphoric Acid Catalysts
}

\author{
Jolene P. Reid and Jonathan M. Goodman* \\ Centre for Molecular Informatics, Department of Chemistry, University of Cambridge, Lensfield Road, Cambridge CB2 1EW, United \\ Kingdom
}

\section{Supporting Information}

ABSTRACT: BINOL-derived phosphoric acids provide effective asymmetric catalysis for many organic reactions. Catalysts based on this scaffold show a large structural diversity, especially in the $3,3^{\prime}$ substituents, and little is known about the molecular requirements for high selectivity. As a result, selection of the best catalyst for a particular transformation requires a trial and error screening process, as the size of the $3,3^{\prime}$ substituents is not simply related to their efficacy: the right choice is neither too large nor too small. We have developed an approach to identify and quantify structural features on the catalyst that determine selectivity. We show that the application of quantitative steric parameters (a new measure, $\operatorname{AREA}(\theta)$, and rotation barrier) to an imine hydrogenation reaction allows the identification of catalyst features necessary for efficient stereoinduction, validated by QM/MM hybrid calculations.

\section{INTRODUCTION}

The discovery of catalysts that efficiently facilitate organic transformations in a stereocontrolled fashion is central to synthetic organic chemistry. ${ }^{1}$ Currently, this process is usually dominated by empirical observations. However, if we can identify and understand general design principles, it should be possible to devise improved or novel catalysts with a high level of confidence. ${ }^{2}$ Recent years have seen the emergence of BINOL-derived phosphoric acids as powerful catalysts for asymmetric transformations. ${ }^{3-5}$ Despite their popularity, most literature reports of reactions do not analyze the detailed origin of stereoinduction. Why are some catalysts more selective than others? Calculation of the transition states reveals key catalystsubstrate interactions that allow efficient transfer of chiral information from catalyst to substrate, assisting catalyst design. ${ }^{2}$ However, this approach is intrinsically time-consuming, as subtle structural changes can have a major impact on enantioselectivity and separate calculations are required for each catalyst. An alternative approach is to discover relationships between catalyst descriptors and enantioselectivity. Such relationships should give rapid insights into the important structural features that are necessary for efficient stereoinduction. $^{6-8}$ Unfortunately, however, it seems that stereoinduction does not simply follow steric bulk or any other readily comprehensible measure of the catalyst.

The selectivity of chiral phosphoric acid catalyzed reactions is dependent on the choice of substituents in the $3,3^{\prime}$ positions of the binaphthol rings: they must be neither too large nor too small, but just right. In this paper, we present a model, validated by DFT studies, which provides insight into the role of $3,3^{\prime}$ groups.

\section{RESULTS AND DISCUSSION}

1. Trends and Steric Descriptors. The structure of the substituents at the $3,3^{\prime}$ positions have a considerable effect on the stereochemical outcome of many reactions, and, usually, large steric bulk is required for high enantioselectivity. However, excessive steric demands in these positions may stop reactions altogether, ${ }^{9}$ or, in some cases, reverse the sense of stereoinduction. ${ }^{10}$ As a result, the optimal choice of substituent and the stereochemical outcome are extremely difficult to predict (Figure 1).

The complete steric description of a system is a demanding task. Computationally screening a large number of substratecatalyst complexes is a computer-intensive process for establishing which properties at an atomic scale determine the enantioselectivity. Alternatively, it may also be possible to develop correlations between catalyst descriptors and enantioselectivity. This method allows exploration of the molecular features affecting the transition state without making any assumptions about the mechanism. A key aspect in developing correlations is identifying appropriate parameters to connect changes in structure with selectivity. The identification of descriptors is facilitated by the observation that large steric bulk

Received: March 17, 2016

Published: May 26, 2016 


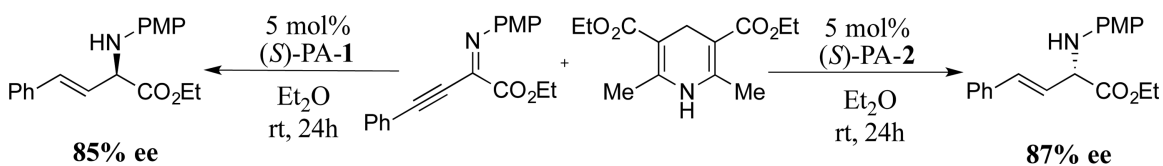

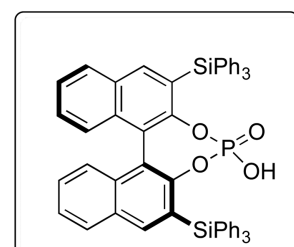

(S)-PA-1

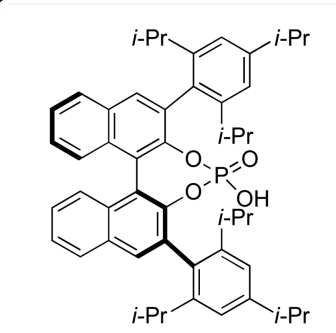

(S)-PA-2

Figure 1. Example reported by You et al. ${ }^{10 \mathrm{~b}}$ demonstrating reaction sensitivity to $3,3^{\prime}$ group.

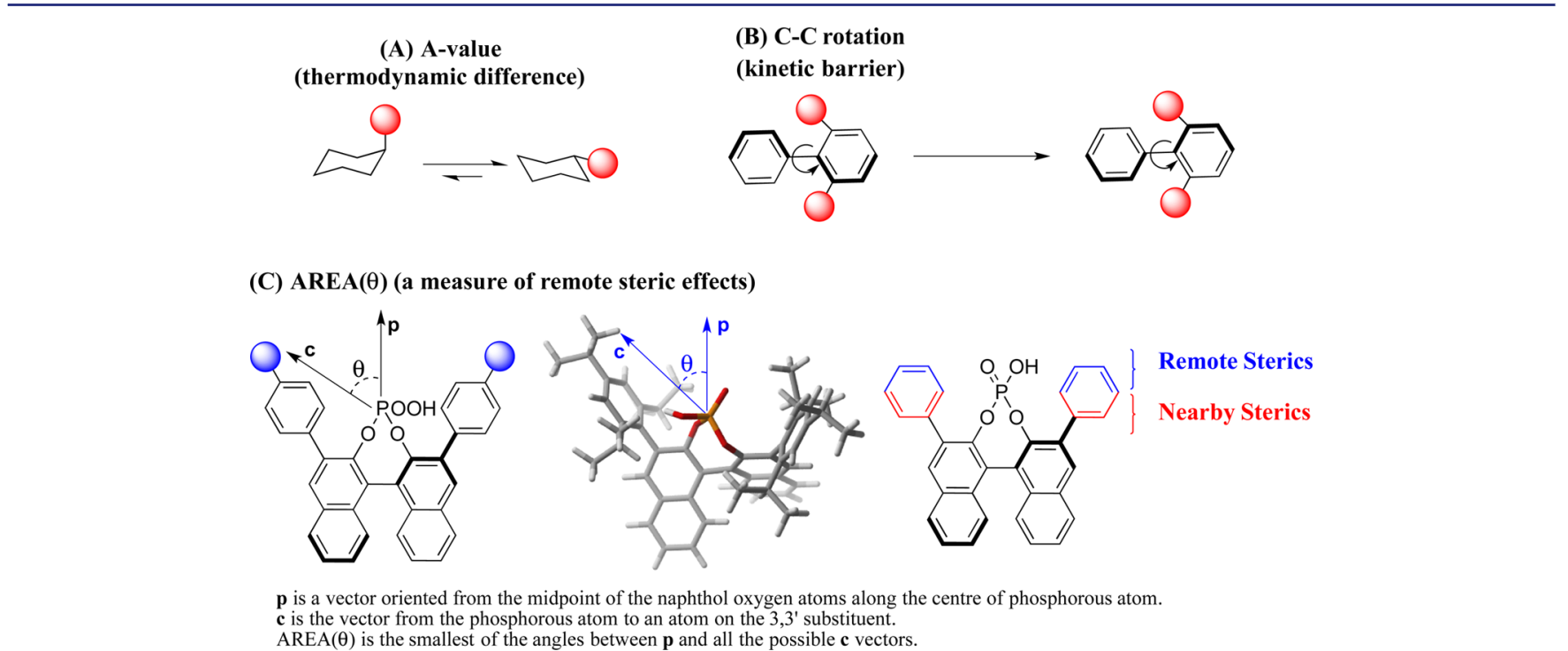

Figure 2. Catalyst steric parameters evaluated in this study. Steric parameters (A) and (B) measure nearby bulk, (C), AREA( $\theta)$, measures steric effects distant from the phosphoric acid moiety. 3D structure of $(S)$-TRIP is shown in a wire frame model as an example. We used the value for $\operatorname{AREA}(\theta)$ from the global minimum, as the value did not vary by more than a degree when conformations within $10 \mathrm{~kJ} \mathrm{~mol}^{-1}$ were considered.

is required for high selectivity. On the basis of this, we began to examine methods in which steric effects could be described numerically. We tried three different measures of steric requirements: (i) $A$-values, a widely used steric parameter, ${ }^{11}$ (ii) rotation barrier for a phenyl group, ${ }^{12,13}$ (iii) A Remote Environment Angle: $\operatorname{AREA}(\theta)$, a measure of space less close to the phosphoric acid group (details in Figures 2 and 4).

A-values are derived from a conformational study on the equilibrium position of ring flipping in monosubstituted cyclohexane rings. The destabilization of the axial conformation is due to 1,3 diaxial interactions. Rotational barriers for a phenyl group are derived from the energy required for rotation around the central $\mathrm{C}-\mathrm{C}$ bond. The interaction between the $\mathrm{R}$ groups and the hydrogens on the opposing aryl ring are responsible for the destabilization of the eclipsed conformation.

Although both $A$-values and rotation barriers can be derived experimentally, we have calculated them to ensure they are available and consistent for all groups of interest. Table 1 gives the approximate values of the energy difference between the equatorial position and the axial, which were calculated using the local Boltzmann-weighted energetic minimum of both the
Table 1. Steric Parameters Calculated Using OPLS-2005 Force Field, ${ }^{14-16}$ in MacroModel (Details in the Supporting Information) ${ }^{17 a}$

\begin{tabular}{lccc}
\multicolumn{1}{c}{ 3,3' group } & $\begin{array}{c}\text { computed } A \text {-value } \\
(\mathrm{A})\end{array}$ & $\begin{array}{c}\text { rotation barrier } \\
(\mathrm{B})\end{array}$ & $\begin{array}{c}\text { AREA }(\theta) \\
(\mathrm{C})\end{array}$ \\
$\mathrm{H}$ & 0.00 & 0.00 & 107 \\
$\mathrm{CHPh}_{2}$ & 1.76 & 1.13 & 47 \\
$\mathrm{SiPh}_{3}$ & 4.85 & 1.35 & 29 \\
$\mathrm{Ph}$ & 4.39 & 2.05 & 70 \\
1-naphth & 4.07 & 13.63 & 62 \\
2-naphth & 4.35 & 2.13 & 49 \\
9-anthryl & 14.40 & 28.31 & 61 \\
9-phenanthryl & 4.09 & 14.45 & 48 \\
4-PhC $\mathrm{H}_{6} \mathrm{H}_{4}$ & 4.35 & 2.01 & 50 \\
4- ${ }^{-} \mathrm{BuC}_{6} \mathrm{H}_{4}$ & 4.37 & 2.02 & 49 \\
3,5-(Ph) ${ }_{2} \mathrm{C}_{6} \mathrm{H}_{3}$ & 4.21 & 2.21 & 36 \\
3,5- $\left(\mathrm{CF}_{3}\right)_{2} \mathrm{C}_{6} \mathrm{H}_{3}$ & 4.03 & 2.02 & 62 \\
2,4,6-(Me ${ }_{3} \mathrm{C}_{6} \mathrm{H}_{2}$ & 15.97 & 21.58 & 61 \\
2,4,6-( $\left.{ }^{(} \mathrm{Pr}\right)_{3} \mathrm{C}_{6} \mathrm{H}_{2}$ & 26.33 & 28.40 & 51
\end{tabular}

${ }^{a}$ All energies in $\mathrm{kcal} \mathrm{mol}^{-1}$. 
axial and equatorial conformations generated from a conformational search in MacroModel. For the groups, the equatorial preference increases with size, although increasing the steric demands remote from the cyclohexane ring is unimportantonly nearby sterics are assessed by this measure (Figure 2). As we expected, $A$-values were connected with stereoselectivity, but, as we also expected, it is clear that they do not tell the complete story (Figure 3). The general agreement (Figure 3,

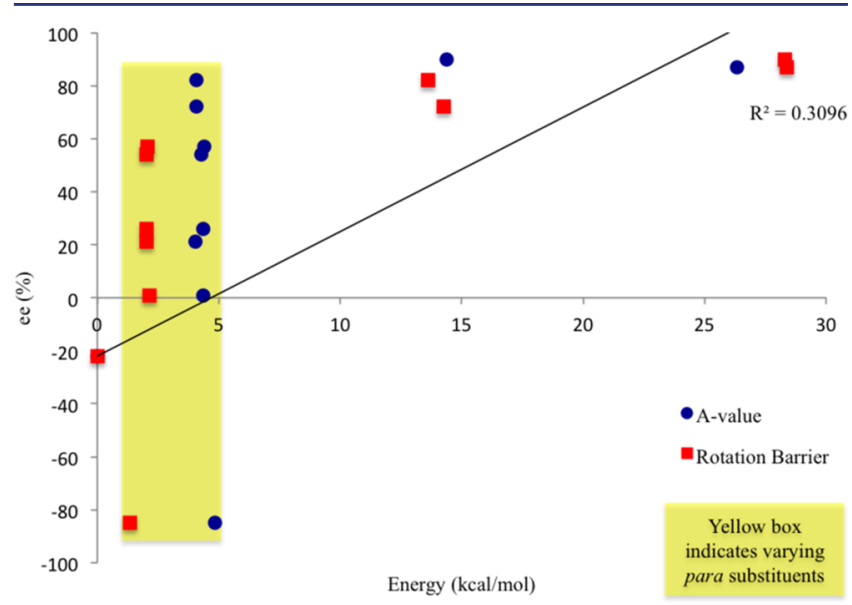

Figure 3. Proximal steric parameters evaluated in this study, literature example reported by You et al. ${ }^{10 \mathrm{~b}}$

circles and squares) between the parameter sets implies that they are both measuring similar properties. The steric bulk distant from the active site, is not taken into account by the $A$ value or the rotational barrier, is a key structural feature affecting enantioselectivity. For example, aromatic rings with hydrogen in the 2,6 positions all have similar A-values (approximately 4.2) and similar rotation barriers (about 2.0). Changing substituents in the 3,4 , and 5 positions does not affect the proximal steric effect but does change the enantioselectivity. Figure 3, therefore, shows a vertical line of points for $A$-values, at about 4.2 , and for rotation barriers at about 2.0. ${ }^{18}$

We therefore sought to develop an alternative steric parameter to describe the remote steric demands of the substituents. Conformational analysis of the phosphoric acid shows the distinctive feature that the active site is deep inside a chiral pocket. What size cone, with its point on the phosphorus atom, would fit inside this cavity? In order to devise a measure of this which is straightforward to calculate, using the global minimum we define the ligand $\operatorname{AREA}(\theta)$ as the smallest angle (in degrees) between the center of an atom located on the $3,3^{\prime}$ group and the vector from center of the phosphorus atom to the midpoint of the binapthol oxygens (Figure 2). Substituents which crowd access to the phosphoric acid, such as 4-tert-butylbenzene, $\operatorname{AREA}(49)$, have smaller $\operatorname{AREA}(\theta)$ values than less sterically demanding substituents, such as phenyl, AREA(70), even though the $A$-value is identical (Table 1). TRIP has a much larger $A$-value than $\mathrm{Ph}_{3} \mathrm{Si}(26.33$ vs 4.85$)$ but is less sterically demanding away from the phosphorus: $\operatorname{AREA}(51)$ vs AREA(29). Computation of this parameter is straightforward, and Figure 4 shows a few examples.

On comparing a series of catalysts for the Mannich reaction, ${ }^{19}$ it was found that the correlation provides a good fit to the experimental data, suggesting that it reflects an underlying physical phenomenon. ${ }^{20}$

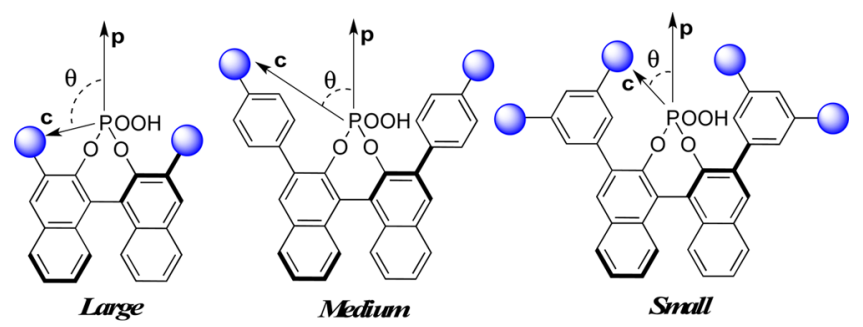

Figure 4. Variation in $\operatorname{AREA}(\theta)$ with $3,3^{\prime}$ substituent.

2. Insight into the Stereochemical Role of the 3,3' Groups. Not all reactions follow such a straightforward pattern, in which the stereoselectivity increases linearly with decreasing $\operatorname{AREA}(\theta)$. For some reactions, the increase stops and then changes to a decline. Arguably the most interesting and mechanistically informative plots are those that are nonlinear. This is shown in the transfer hydrogenation reaction reported by You et al. ${ }^{10 \mathrm{~b}}$ Experimentally You et al. found that changing the 3,3' groups from 2,4,6-triisopropylphenyl $($ medium $\operatorname{AREA}(\theta))$ to $\mathrm{SiPh}_{3}(\operatorname{small} \operatorname{AREA}(\theta))$ inverted the sense of stereoinduction (Figure 6). However, it is not clear how the $3,3^{\prime}$ group induces such a strong reversal.
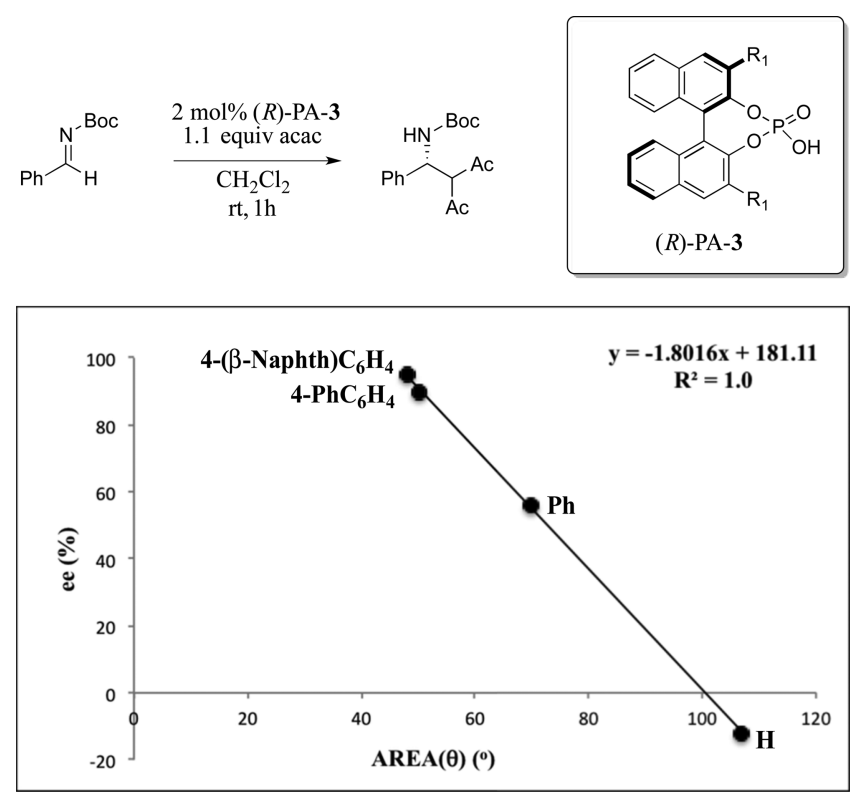

Figure 5. Ligand $\operatorname{AREA}(\theta)$ parameter provides a linear relationship with enantioselectivity in Terada's Mannich reaction. ${ }^{19}$

The mechanism of this class of reactions is well established. ${ }^{21}$ However, the break in linearity indicates some unexpected feature in the enantiodetermining step. To probe this feature we decided to investigate the change in mechanism computationally using high-level ONIOM calculations, which we have previously applied to similar systems. ${ }^{21}$ Slightly simplified molecules were used in the calculations: PMP group was replaced by a $\mathrm{Ph}$; the dimethyl Hantzsch ester was used instead of the diethyl counterpart. The imine can orient itself with respect to the catalyst in one of two ways. Noting that the $C_{2}$ symmetry of the phosphate anion allows us to draw the imine at the front of the diagram without loss of generality, the $\mathrm{N}$ substituent, $R_{1}$, can be directed away from the front $3,3^{\prime}$ group, which we call Type $I$, or toward the $3,3^{\prime}$ groups, which we call 


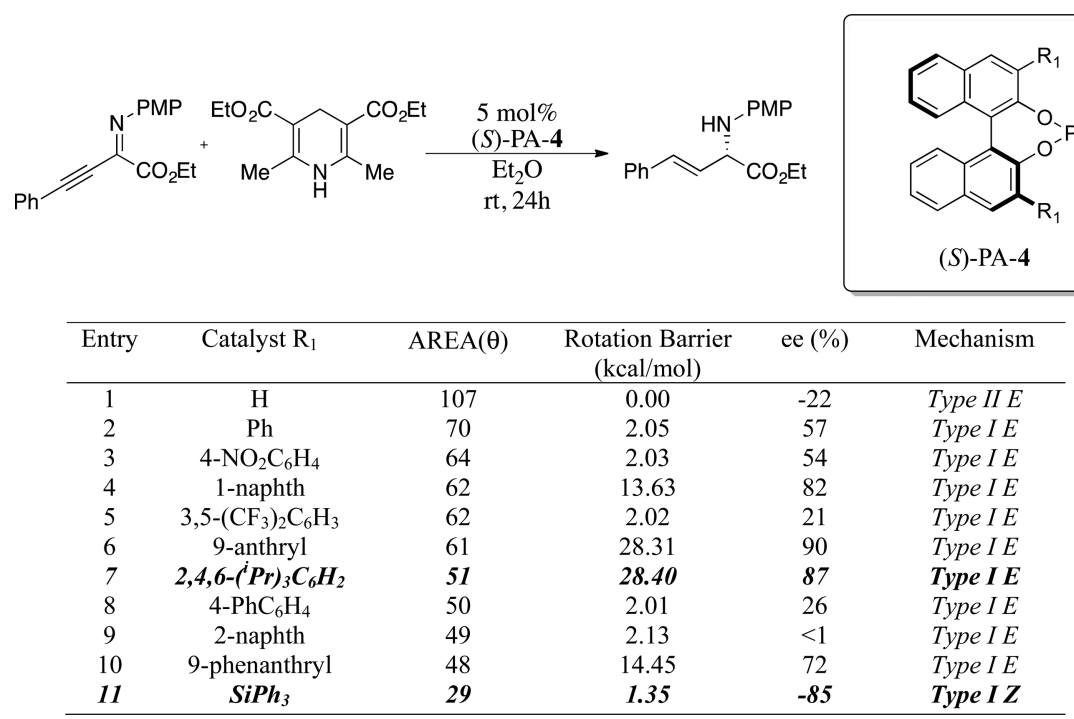

Figure 6. Break in linearity obtained for You's transfer hydrogenation. Type I and Type II are explained in Figure 7.

Type II. Additionally, the imine can exist as either the $E$ or $Z$ stereoisomer; we define $E$ or $Z$ based on steric size. ${ }^{22}$ Combining these considerations, four unique TSs for the transfer hydrogenation reaction are formulated. The possible transition states and the products they afford are given below (Figure 7).

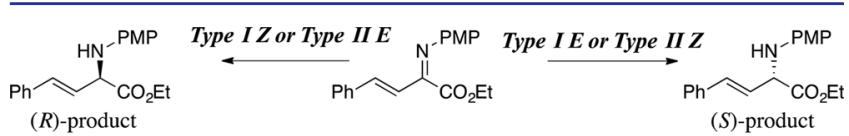

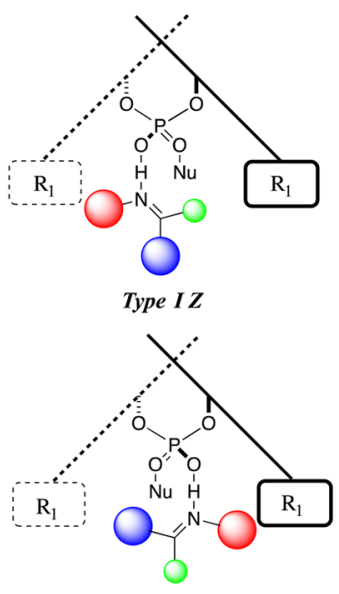

Type II E
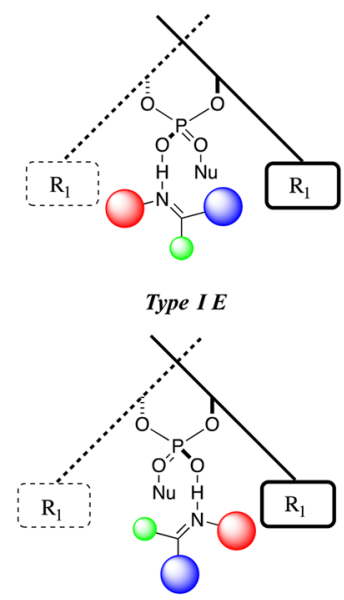

Type II Z

Figure 7. Transition state models for the prediction of enantioselective outcome.

Transition states for the full catalyst system were located using ONIOM (B3LYP/6-31G**: UFF), single-point energies M06-2X/6-31G** were then calculated for the low energy reaction pathways (see the Supporting Information for further details). Solvation free energy corrections were computed by means of the PCM model, the results are summarized in Figures 8 and 9. For the TRIP catalyzed reaction the lowest calculated transition state is TS2-E (Type I E), which is in good agreement with the experimentally observed outcome (computed ee 99\%). For the $\mathrm{SiPh}_{3}$ derived phosphoric acid catalyzed reaction, the lowest energy transition state is TS4-Z (Type I Z), which accounts for the experimentally observed reversal in stereoinduction. Although the calculated energy values are lower than compared to experiment (computed ee $-40 \%$ ), the reproduction of qualitative trends is accurately predicted. The ONIOM (M06-2X/6-31G**:UFF) method indicates that the discrepancy between calculations and experiment traces back to the B3LYP component of the optimization. ONIOM (M06$\left.2 \mathrm{X} / 6-31 \mathrm{G}^{* *}: \mathrm{UFF}\right)$ gave a larger preference for the Type $I Z$ pathway than ONIOM (B3LYP/6-31G**:UFF). The energy difference between TS4- $Z$ and TS4- $E$ re-evaluated using ONIOM (M06-2X/6-31G**:UFF) was calculated to be 2.9 $\mathrm{kcal} / \mathrm{mol}$. Although the B3LYP geometries are generally found to be reliable, ${ }^{23}$ this example was a difficult case. The Supporting Information provides a comparison of energy differences between the TSs in Figures 8 and 9 computed using both ONIOM methods. The combination of calculations and experimental data provides compelling evidence that the imine stereoisomer involved in the transition state changes within a catalyst screen. For both catalysts, the Type II pathways were higher in energy due to the additional steric interactions and the directly competing one was that of the opposite imine conformation. Catalysts with very large $\operatorname{AREA}(\theta)$ have little proximal bulk, and these substituents cannot differentiate between Type I and Type II pathways. In these cases, low enantioselectivities are obtained and either pathway can be slightly favored (Figures 5 and 6). Visual inspection of the Type II TS's for the TRIP-catalyzed reactions show the N-substituent is directed toward the proximal isopropyl groups, having little interaction with the one remote from the phosphoric acid moiety. This implies that the bulk proximal to the phosphoric acid moiety controls Type I vs Type II selectivity. This serves to explain the discrepancies in selectivity between catalysts with similar distal bulk, which we quantify using $\operatorname{AREA}(\theta)$, but varying proximal bulk (Figure 6, entries 7 and 8). This physical factor is not explicitly accounted for by the ligand $\operatorname{AREA}(\theta)$ but can be described by a proximal bulk steric parameter, such as the rotation barrier (Table 1, although both proximal bulk steric parameters seem to underestimate the nearby steric effects of $\mathrm{SiPh}_{3}$ ). The reversal in lowest TS observation can be rationalized by our qualitative model Figure 10: facilitating the reaction in a medium catalyst cavity results in the $E$ 


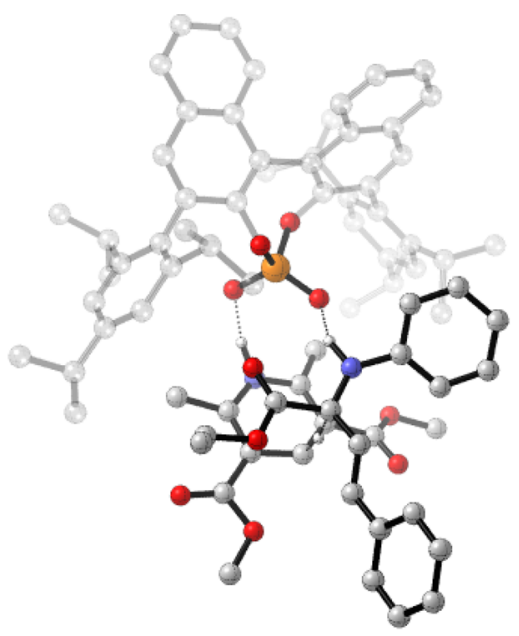

TS1-Z: $\Delta \Delta \mathrm{G}^{\ddagger}=+8.6 \mathrm{kcal} \mathrm{mol}^{-1}, \Delta \Delta \mathrm{G}_{\mathrm{sol}}{ }^{\ddagger}=+10.3 \mathrm{kcal} \mathrm{mol}^{-1}$

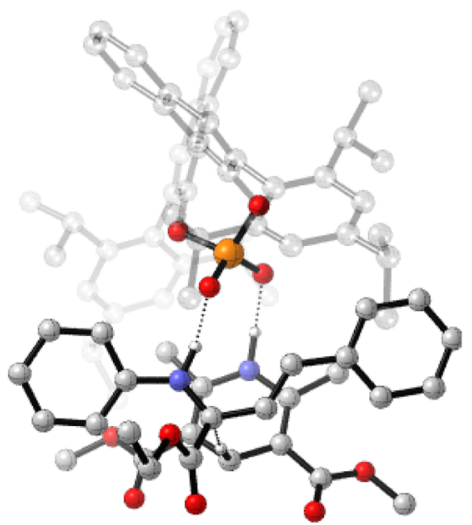

TS2- $E: \Delta \Delta \mathrm{G}^{\ddagger}=0 \mathrm{kcal} \mathrm{mol}^{-1}, \Delta \Delta \mathrm{G}_{\mathrm{sol}}^{\ddagger}=0 \mathrm{kcal} \mathrm{mol}^{-1}$

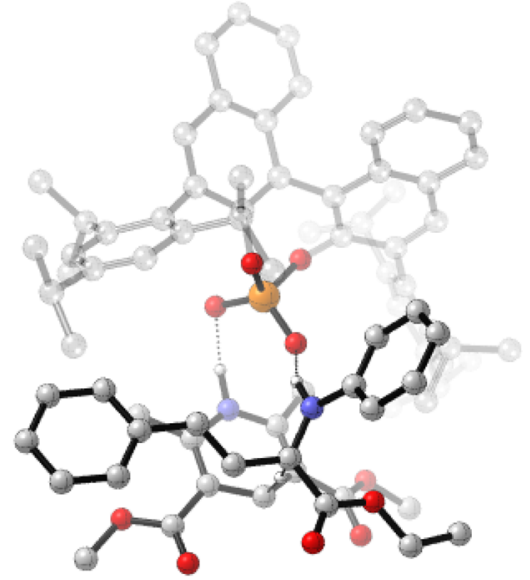

TS1-E: $\Delta \Delta \mathrm{G}^{\ddagger}=+9.5 \mathrm{kcal} \mathrm{mol}^{-1}, \Delta \Delta \mathrm{G}_{\mathrm{sol}}{ }^{\ddagger}=+9.0 \mathrm{kcal} \mathrm{mol}^{-1}$

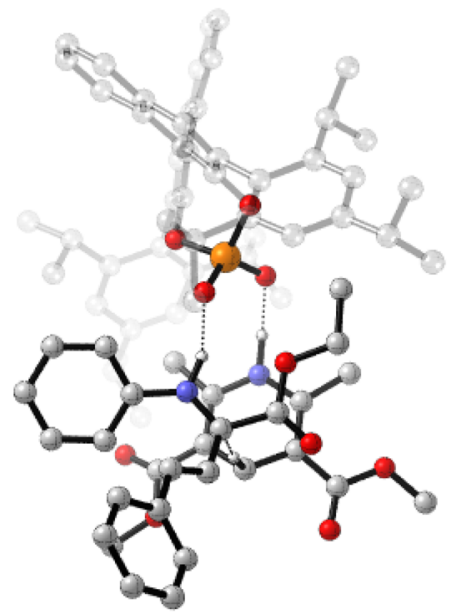

TS2-Z: $\Delta \Delta \mathrm{G}^{\ddagger}=+3.2 \mathrm{kcal} \mathrm{mol}^{-1}, \Delta \Delta \mathrm{G}_{\mathrm{sol}}^{\ddagger}=+3.5 \mathrm{kcal} \mathrm{mol}^{-1}$

Figure 8. Competing TSs for the TRIP catalyzed transfer hydrogenation reaction. ONIOM (B3LYP/6-31G**: UFF), single-point energy M06-2X/ $6-31 \mathrm{G}^{* *}$. Grayed-out regions were treated with $\mathrm{UFF}$, and the full-color regions were treated $\mathrm{B} 3 \mathrm{LYP} / 6-31 \mathrm{G}^{* *}$. Structures generated using CYLview. $^{24}$

conformation being favored; this conformation reduces the internal substrate steric interactions present in the $Z$ conformation. However, as the catalyst cavity decreases, the interaction between the $\mathrm{R}$ groups and the $3,3^{\prime}$ groups becomes more energetically costly than the internal steric repulsion and so the $Z$ transition state is favored. The imine $\mathrm{R}$ groups are directed toward the bulk distant from the catalyst, it is this interaction that controls which stereoisomer of the imine is present in the TS.

This model implies that similar reactions proceeding via Type I $E$ pathways will not be suitable for a reaction catalyzed by small ligand $\operatorname{AREA}(\theta)$ catalysts. Such catalysts bias toward a $Z$ transition state leading to little stereoinduction. An optimal catalyst for such a reaction; would be one that is neither too big (raises $E$ relative to $Z$ ) nor one that is too small (cannot differentiate between Type I or Type II) but somewhere in between. In examples, where small ligand $\operatorname{AREA}(\theta)$ catalysts proceed with high levels of enantioslectivity the favored pathway would be a Type $I Z$. Examples where this general trend is observed are given in Table 2. However, without detailed mechanistic investigations only a correlation can be established. None the less, the general agreement with our model and experimental enantioselectivity trends indicates such an approach can be used to guide strategic choice of catalyst based on substrate structures and reaction pathway. We are now studying the origin of nonlinear effects in other reactions in the hope of developing a more comprehensive predictive model to aid optimal catalyst choice.

\section{CONCLUSIONS}

In conclusion, the steric features of the $3,3^{\prime}$ groups on the phosphoric acid that determine stereochemical induction has been investigated. Product selectivity in these reactions is dependent on both sterics distant from the active site, quantified by the ligand $\operatorname{AREA}(\theta)$ and proximal sterics, quantified by the rotation barrier. Applying the steric parameters to a validating case study: the hydrogenation of imines suggested that both proximal and remote sterics played important roles. This prompted a mechanistic study by QM/ MM. Our calculations suggest that proximal bulk controls the orientation of the imine (Type I vs Type II) and the remote sterics control the imine stereoisomer ( $E$ vs $Z$ ) present. This 


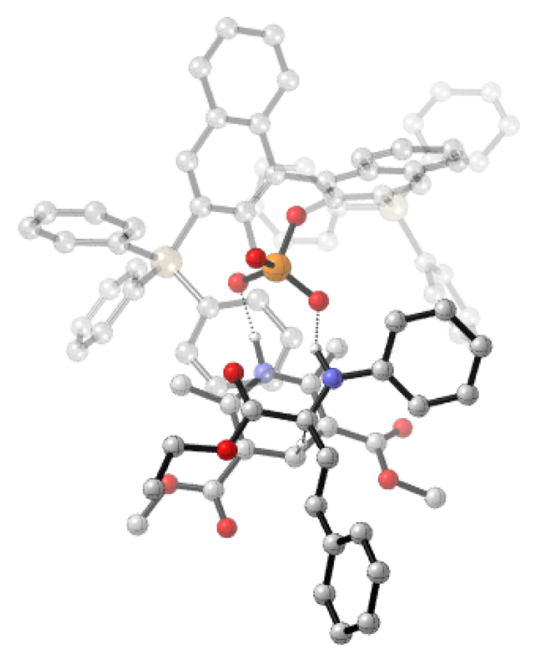

TS3-Z: $\Delta \Delta \mathbf{G}^{\ddagger}=+8.2 \mathrm{kcal} \mathrm{mol}^{-1}, \Delta \Delta \mathbf{G}_{\text {sol }}{ }^{\ddagger}=+8.0 \mathrm{kcal} \mathrm{mol}^{-1}$

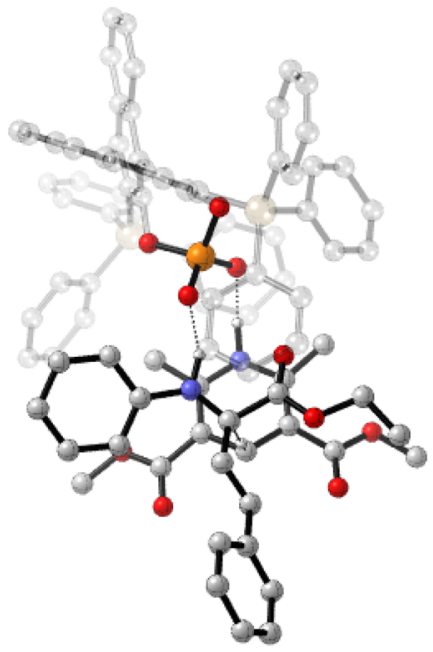

TS4-Z: $\Delta \Delta \mathrm{G}^{\ddagger}=0 \mathrm{kcal} \mathrm{mol}^{-1}, \Delta \Delta \mathrm{G}_{\mathrm{sol}}^{\ddagger}=0 \mathrm{kcal} \mathrm{mol}^{-1}$

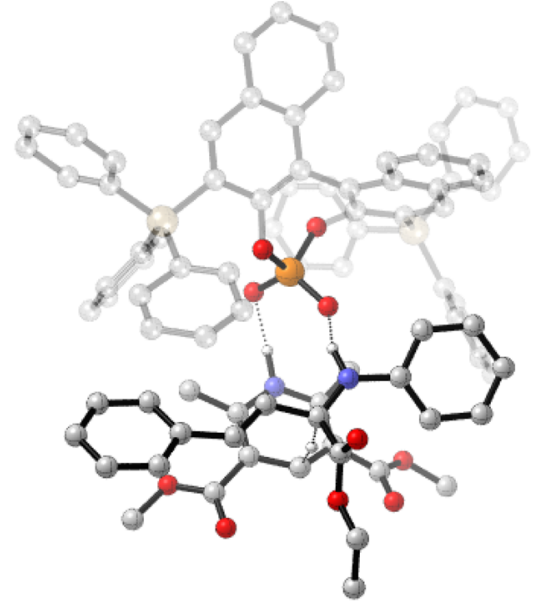

TS3-E: $\Delta \Delta \mathbf{G}^{\ddagger}=+10.8 \mathrm{kcal} \mathrm{mol}^{-1}, \Delta \Delta \mathrm{G}_{\mathrm{sol}}^{\ddagger}=+9.9 \mathrm{kcal} \mathrm{mol}^{-1}$

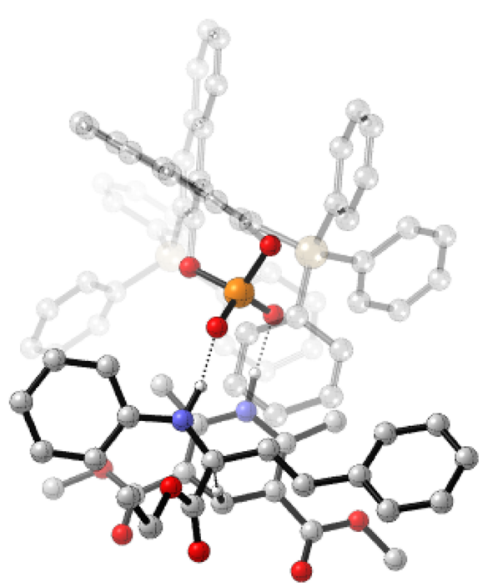

TS4-E: $\Delta \Delta \mathrm{G}^{\ddagger}=+0.5 \mathrm{kcal} \mathrm{mol}^{-1}, \Delta \Delta \mathrm{G}_{\mathrm{sol}}^{\ddagger}=-0.7 \mathrm{kcal} \mathrm{mol}^{-1}$

Figure 9. Competing TSs for the $\mathrm{SiPh}_{3}$ catalyzed transfer hydrogenation reaction. ONIOM (B3LYP/6-31G**: UFF), single-point energy M06-2X/ 6-31G**. Grayed-out regions were treated with UFF, and the full-color regions were treated B3LYP/6-31G**. Structures generated using CYLview. ${ }^{24}$

information was applied in developing a qualitative model that logically describes the change in transition state preference with $3,3^{\prime}$ substituents. Applying the qualitative model to similar systems can rationalize selectivity trends with varying $3,3^{\prime}$ substituent, in which high selectivity's with medium $\operatorname{AREA}(\theta)$ catalysts are obtained for Type I E pathways, indicating that this study could be used as a guide for catalyst selection for a given transformation based on reaction pathway. Additionally, our method allows prediction of catalyst properties through computation, which could promote the identification of new and improved catalysts. We are now studying the origin of nonlinear effects in other reactions in the hope of developing a more comprehensive predictive model to aid optimal catalyst choice.

\section{COMPUTATIONAL METHODS}

For the $A$-value calculation, the local Boltzmann weighted energetic minimum of both the axial and equatorial conformations were calculated using MacroModel (version 9.9). ${ }^{17}$ MacroModel calculated the energies and geometries according to the OPLS-2005 force field. ${ }^{14-16}$
The energy barrier for rotation was calculated from torsional energy profiles generated by MacroModel (version 9.9), ${ }^{17}$ using the OPLS2005 force field. ${ }^{14-16}$

The ligand $\operatorname{AREA}(\theta)$ was calculated on the lowest energy conformer, generated from a conformational search in MarcoModel (version 9.9), ${ }^{17}$ according to the OPLS-2005 force field. ${ }^{14-16}$ For vector assignment, see Figure 2.

For the QM/MM hybrid calculations on the full catalyst, transition states were located first, by a conformational search in MacroModel (version 9.9) $)^{17}$ using the OPLS-2005 force field. ${ }^{14-16}$ Those conformers below $10 \mathrm{~kJ} \mathrm{~mol}^{-1}$ of the minimum were optimized using the ONIOM method implemented in Gaussian 03 (revision E.01). ${ }^{29}$ The B3LYP density functional, ${ }^{30,31}$ and split-valence polarized $6-31 \mathrm{G}^{* *}$ basis set, ${ }^{32,33}$ were used for the high-layer, and the force field $\mathrm{UFF},{ }^{34}$ was used for the low-layer. The reactants and the phosphoric acid moiety of the catalyst were included in the high-layer, and the remaining regions of the catalyst were treated as the low-layer. This method has previously been shown to give excellent results when used to describe reactions catalyzed by chiral phosphoric acids. ${ }^{21,22,35-39}$ The position of the partition within the catalyst was chosen as the phosphoric acid binds directly to the reagents, whereas the remaining catalyst acts as steric bulk and can be adequately described by molecular mechanics. ${ }^{21}$ Single point energy calculations were 

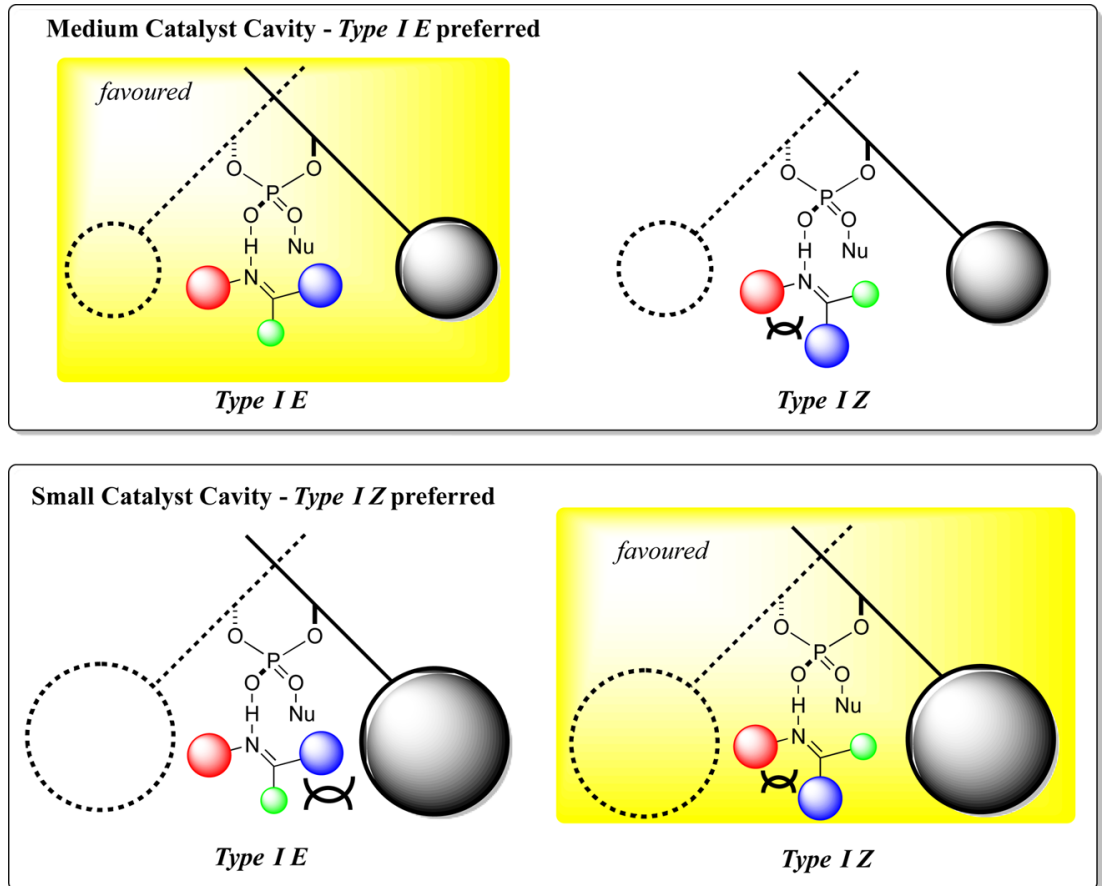

Figure 10. Qualitative model describing the preference for $Z$ transition state with small ligand angle catalysts. In the $E$ configuration, the largest $\mathrm{R}$ groups are coplanar and so internal steric interactions are minimized. The $Z$ imine conformation allows placing two groups distant from the majority of the catalyst bulk, but this is offset by their proximity to each other.

Table 2. Examples of Nucleophilic Additions to Imines Catalyzed by Chiral Phosphoric Acids

\begin{tabular}{|c|c|c|c|c|}
\hline reaction & mechanism & $\operatorname{AREA}(\theta)>70(\mathrm{ee} \%)$ & medium $\operatorname{AREA}(\theta)(\mathrm{ee} \%)$ & $\operatorname{AREA}(\theta)<36(\mathrm{ee} \%)$ \\
\hline peroxidation of imines $^{25}$ & Type I E & & 84 & 3 \\
\hline addition of thiols ${ }^{26}$ & Type I E & & 91 & 11 \\
\hline Strecker reaction $^{27}$ & Type I E & & 84 & 0 \\
\hline reductive amination ${ }^{28}$ & Type I Z & 7 & 65 & 87 \\
\hline
\end{tabular}

performed on the resulting structures using M06-2X density functional,,$^{40}$ and the $6-31 \mathrm{G}^{* *}$ basis set, ${ }^{30,31}$ using nondefault convergence criteria (fine grid density, ultrafine accuracy level) as implemented in the Jaguar program (version 7.9). ${ }^{41}$ This energy was used to correct the gas-phase energy derived from the ONIOM calculations. Free energies in solution were derived from structures optimized in the gas phase at the ONIOM (B3LYP/6-31G**:UFF), level of theory by means of a single point calculation using M06-2X/6$31 \mathrm{G}^{* *}$ with the polarizable continuum model (PCM) as implemented in the Jaguar program (version 7.9), using diethyl ether (probe radius $=2.74 \AA$ ) as the solvent. ${ }^{42}$ These values were used to correct the Gibbs free energy derived from the ONIOM calculations.

To further validate the results, the lowest energy transition state conformation from this process was reoptimized using the ONIOM method implemented in Gaussian 09 (revision D.01), ${ }^{43}$ M06-2X/6$31 \mathrm{G}^{* *}$ was used for the high-layer, and the force field UFF was used for the low-layer. The free energies obtained from this process were corrected by a single point calculation in the same manner as above.

\section{ASSOCIATED CONTENT}

\section{S Supporting Information}

The Supporting Information is available free of charge on the ACS Publications website at DOI: 10.1021/jacs.6b02825. According to the University of Cambridge data management policy, all the data used in this paper is available either in the paper or in the Supporting Information. A copy of the data is also available in the University of Cambridge repository at https://www.repository.cam.ac.uk/.
Complete list of authors in the Gaussian03 and Gaussian09 references, Cartesian coordinates of all the catalyst structures, Cartesian coordinates, energies, and values of imaginary frequencies of all the transition state structures (PDF)

\section{AUTHOR INFORMATION}

\section{Corresponding Author}

*jmg11@cam.ac.uk

Notes

The authors declare no competing financial interest.

\section{ACKNOWLEDGMENTS}

We thank EPSRC for funding.

\section{REFERENCES}

(1) Noyori, R. Angew. Chem., Int. Ed. 2002, 41, 2008.

(2) Houk, K. N.; Cheong, P. H.-Y. Nature 2008, 455, 309.

(3) Akiyama, T. Chem. Rev. 2007, 107, 5744.

(4) Terada, M. Synthesis 2010, 2010, 1929.

(5) Parmar, D.; Sugiono, E.; Raja, S.; Rueping, M. Chem. Rev. 2014, 114, 9047.

(6) Harper, K. C.; Bess, E. N.; Sigman, M. S. Nat. Chem. 2012, 4, 366.

(7) Harper, K.; Sigman, M. S. J. Org. Chem. 2013, 78, 2813.

(8) Milo, A.; Neel, A. J.; Toste, F. D.; Sigman, M. S. Science 2015, 347, 737. 
(9) For examples where no reaction is observed for structurally large catalysts, see: (a) Saha, S.; Schneider, C. Chem. - Eur. J. 2015, 21, 2348. (b) Lu, M.; Lu, Y.; Zhu, D.; Zeng, X.; Li, X.; Zhong, G. Angew. Chem., Int. Ed. 2010, 49, 8588. (c) Wen, W.; Zeng, Y.; Peng, L.-Y.; Fu, L.-N.; Guo, Q.-X. Org. Lett. 2015, 17, 3922.

(10) For examples where large catalysts proceed with reversal in stereoinduction, see: (a) Yu, X.; Wang, Y.; Wu, G.; Song, H.; Zhou, Z.; Tang, C. Eur. J. Org. Chem. 2011, 2011, 3060. (b) Kang, Q.; Zhao, Z.A.; You, S.-L. Org. Lett. 2008, 10, 2031. (c) Gu, Q.; Rong, Z.-Q; Zheng, C.; You, S.-L. J. Am. Chem. Soc. 2010, 132, 4056. (d) Liao, S.;

Coric, I.; Wang, Q.; List, B. J. Am. Chem. Soc. 2012, 134, 10765.

(e) Feng, J.; Yan, W.; Wang, D.; Li, P.; Sun, Q.; Wang, R. Chem. Commun. 2012, 48, 8003.

(11) Winstein, S.; Holness, N. J. J. Am. Chem. Soc. 1955, 77, 5562.

(12) Bott, G.; Field, L. D.; Sternhell, S. J. Am. Chem. Soc. 1980, 102, 5618.

(13) Adams, R.; Yuan, H. C. Chem. Rev. 1933, 12, 261.

(14) Kaminski, G. A.; Friesner, R. A.; Tirado-Rives, J.; Jorgensen, E. J. Phys. Chem. B 2001, 105, 6474.

(15) Jorgensen, W. L.; Maxwell, D. S.; Tirado-Rives, J. J. Am. Chem. Soc. 1996, 118, 11225.

(16) Jorgensen, W. L.; Tirado-Rives, J. J. Am. Chem. Soc. 1988, 110, 1657.

(17) MacroModel, version 9.9; Schrodinger, LLC: New York, 2009.

(18) The molecular descriptors can also be compared to $\Delta \Delta G^{\ddagger}$ which gives a similar pattern and no additional insight.

(19) Uraguchi, D.; Terada, M. J. Am. Chem. Soc. 2004, 126, 5356.

(20) We also considered the solvent accessible surface area of the phosphate oxygens as a descriptor. However, the four catalysts have very similar accessible areas, and no correlation could be established.

(21) Simón, L.; Goodman, J. M. J. Am. Chem. Soc. 2008, 130, 8741.

(22) Simón, L.; Goodman, J. M. J. Org. Chem. 2011, 76, 1775.

(23) Wodrich, M. D.; Corminboeuf, C.; Schleyer, P. v. R. Org. Lett. 2006, 8, 3631.

(24) Legault, C. Y. CYLview, 1.0b; Université de Sherbrooke: Sherbrooke, Canada, 2009 (http://www.cylview.org).

(25) Zheng, W.; Wojtas, L.; Antilla, J. C. Angew. Chem., Int. Ed. 2010, $49,6589$.

(26) Ingle, G. K.; Mormino, M. G.; Wojtas, L.; Antilla, J. C. Org. Lett. 2011, 13, 4822.

(27) Rueping, M.; Sugiono, E.; Azap, C. Angew. Chem., Int. Ed. 2006, 45, 2617.

(28) Storer, R. I.; Carrera, D. E.; Ni, Y.; MacMillan, D. W. C. J. Am. Chem. Soc. 2006, 128, 84.

(29) Frisch, M. J. et al. Gaussian 03, Revision E.01; Gaussian, Inc.: Wallingford, CT, 2004.

(30) Becke, A. D. Phys. Rev. A: At., Mol., Opt. Phys. 1988, 38, 3098.

(31) Lee, C.; Yang, W.; Parr, R. G. Phys. Rev. B: Condens. Matter Mater. Phys. 1988, 37, 785.

(32) Krishnan, R.; Binkley, J. S.; Seeger, R.; Pople, J. A. J. Chem. Phys. 1980, 72, 650.

(33) Gill, P. M. W.; Johnson, B. G.; Pople, J. A.; Frisch, M. J. Chem. Phys. Lett. 1992, 197, 499.

(34) Rappe, A. K.; Casewit, C. J.; Colwell, K. S.; Goddard, W. A.; Skiff, W. M. J. Am. Chem. Soc. 1992, 114, 10024.

(35) Simón, L.; Goodman, J. M. J. Am. Chem. Soc. 2009, 131, 4070.

(36) Simón, L.; Goodman, J. M. J. Org. Chem. 2010, 75, 589.

(37) Grayson, M. N.; Pellegrinet, S. C.; Goodman, J. M. J. Am. Chem. Soc. 2012, 134, 2716.

(38) Grayson, M. N.; Goodman, J. M. J. Am. Chem. Soc. 2013, 135, 6142.

(39) Overvoorde, L. M.; Grayson, M. N.; Luo, Y.; Goodman, J. M. J. Org. Chem. 2015, 80, 2634.

(40) Zhao, Y.; Truhlar, D. Theor. Chem. Acc. 2008, 120, 215.

(41) Jaguar, version 7.9; Schrodinger, LLC: New York, 2012.

(42) Mennucci, B.; Tomasi, J. J. Chem. Phys. 1997, 106, 5151.

(43) Frisch, M. J. et al. Gaussian 09, Revision D.01; Gaussian, Inc.: Wallingford, CT, 2013. 